\title{
Linked color imaging improves detection of minimal change esophagitis in non-erosive reflux esophagitis patients
}

\section{(잉 $\odot$}

\author{
Authors \\ Pei Deng*, Min Min", Tenghui Dong, Yiliang Bi, Airong Tang, Yan Liu
}

Institution

Department of Gastroenterology and Hepatology, Affiliated Hospital of Academy of Military Medical Sciences, Beijing, China

submitted 14.11.2017

accepted after revision 14.3.2018

Bibliography

DOI https://doi.org/10.1055/a-0602-3997 |

Endoscopy International Open 2019; 06: E1177-E1183

(c) Georg Thieme Verlag KG Stuttgart · New York

ISSN 2364-3722

Corresponding author

Professor Yan Liu, Department of Gastroenterology and

Hepatology, Affiliated Hospital of Academy of Military

Medical Sciences, Beijing, 100071, China

Fax: +86-10-66947473

13911798288@163.com

\section{ABSTRACT}

Background and study aims Non-erosive reflux disease (NERD) includes minimal change esophagitis (MCE) and no endoscopic abnormalities. However, for most endoscopists, it is difficult to detect MCE with conventional white-light endoscopy (WLE). Linked color imaging ( $\mathrm{LCl}$ ) technology is the most recently developed image-enhancing technology and improves detection and differentiation of subtle muco- sal changes using a color contrast method. This study assessed the efficacy of WLE combined with LCI for diagnosing MCE compared with WLE.

Patients and methods Between February and May 2017, 44 NERD patients and 40 healthy subjects were enrolled in our study. First, the distal esophagus was examined using WLE followed by LCI. Second, three experienced endoscopists observed all the patients' white-light (WL) images and corresponding images of $\mathrm{WL}$ and $\mathrm{LCl}$ and then recorded presence or absence of minimal change esophagitis (MCE $+/-)$. The proportion of minimal change between the two groups was then compared. Third, five blinded endoscopists with different levels of endoscopic experience assessed whether MCE was present. Intraobserver reproducibility and interobserver agreement were described using the kappa value.

Results The proportion of MCE in the NERD group (70.8\%, $35 / 48)$ was higher than that in the control group $(22.5 \%$, $9 / 40, P<0.001)$ when diagnosed by the three experienced endoscopists. Detection rates for MCE using WLE combined with LCI were higher than those using WLE $(43 / 88,48.9 \%$ vs. $29 / 88,33.0 \%, P<0.001)$. With WLE combined with $\mathrm{LCl}$, intraobserver reproducibility significantly improved, indicating that the combined approach can improve interobserver agreement compared with using WLE alone.

Conclusions Endoscopic diagnosis of MCE using WLE combined with $\mathrm{LCl}$ images is effective. Intraobserver reproducibility and interobserver agreement in MCE can be improved when $\mathrm{LCl}$ is applied with conventional imaging (Clinical trial registration number: NCT03068572).

\section{Introduction}

Non-erosive reflux disease (NERD) is defined by presence of troublesome reflux-associated symptoms and absence of mucosal breaks on endoscopy [1]. However, upon careful analysis, the majority of NERD patients did not have completely normal endoscopic findings and were found to have subtle distal esophageal mucosal changes from acid refluxate. These mucosal changes are referred to as minimal change esophagitis

\footnotetext{
* Drs. Deng and Min: These authors contributed equally.
}

(MCE), which is characterized by edema of the mucosal folds, reddish or whitish colors, blurring with friability of the mucosal junction, increased vascularity and microerosion [2-3]. Some experts have reported that superficial and small esophageal lesions in NERD patients that cannot be visualized by conventional white-light endoscopy (WLE) are possible [4]. However, with the extensive evolution of endoscopic technologies, image resolution is increasing; thus, incidence of MCE identification seems to be gradually increasing. Therefore, modification of the conventional Los Angeles classification was proposed to in- 
corporate MCE by adding the grades $\mathrm{N}$ (normal mucosa) and $\mathrm{M}$ (presence of lesions) listed in Japan [5, 6].

Recently, a new LASERO system (FUJIFILM Co., Tokyo, Japan) was developed. This novel technology can enable four types of imaging, namely, white light, linked color imaging ( $\mathrm{LCl}$ ), flexible spectral imaging color enhancement (FICE) and blue laser imaging (BLI). LCl acquires images by simultaneously using narrowband short-wave-length light and white light (WL) in an appropriate balance [7]. LCl-acquired color information is reallocated to differentiate colors that are similar to the mucosal color, resulting in improved performance in depicting blood vessels, and additional image processing enhances color separation for red color, which allows for clear visualization of red blood vessels and white pits [8].

Currently, $\mathrm{LCl}$ is intended to enhance slight color differences in the red region of the mucosa [9]. Thus, $\mathrm{LCl}$ can reveal minimal change lesions much more clearly, which cannot be identified by WL, image-enhanced endoscopy (IEE) or other light sources. Until now, there have been no reports of the endoscopic diagnosis of MCE using $\mathrm{LCl}$. Therefore, this study was conducted to assess the effectiveness of $\mathrm{LCl}$ for diagnosing MCE compared with that of conventional WLE as the primary outcome. The secondary outcome was inter- and intraobserver variation in use of $\mathrm{LCl}$ between endoscopists with different levels of experience.

\section{Patients and methods}

\section{Patients}

This study was performed between February and May 2017. A total of 254 consecutive outpatients were invited to participate and signed an informed consent form. The Institutional Review Board of the Affiliated Hospital of Academy of Military Medical Sciences approved the study protocol, which was registered at ClinicalTrials.gov (study number: NCT03068572). All patients were asked questions from a standardized questionnaire (GerdQ) [10] by an independent person and were enrolled if they met the following inclusion criteria: 1 ) age >18 years; 2) ability to provide written informed consent and undergo an upper endoscopy; and 3) more than 3 reflux episodes/week, absence of mucosal breaks on conventional endoscopy, and intake of standard or double doses of an oral proton pump inhibitor (PPI) for 2 weeks to determine the efficacy of anti-secretory therapy (the so-called PPI test). The PPI test was considered positive when more than $50 \%$ improvement in gastroesophageal reflux disease (GERD) symptoms was observed at the followup visit [11]. Participants who responded to PPI treatment comprised the NERD group. Exclusion criteria: 1) erosive esophagitis or diagnosis of erosive esophagitis within the previous 6 months; 2) presence of columnar lined epithelium; 3) evidence of cancer or mass lesions in the esophagus or gastric lesions (ulcer, polyp, cancer); 4) previous gastrointestinal surgery; 5) esophageal strictures; 6 ) chronic use of medications known to affect esophageal motility; 7) presence of systemic diseases that may interfere with esophageal motility; and 8) use of PPIs or histamine 2-receptor antagonists in the past 4 weeks.
Gender-matched asymptomatic subjects were recruited from a pool of individuals who had undergone endoscopy solely for the purpose of a health check-up during the study period. Just prior to endoscopic examination, all subjects were asked whether they had upper gastrointestinal symptoms in the past 6 months by an independent observer. Exclusion criteria were the same as those for the NERD group.

Baseline characteristics at enrollment, including sex, age, height, weight, and smoking, were recorded on the GerdQ questionnaire for GERD symptom assessment. The GerdQ was administered before endoscopy; however, the patients were not aware of the endoscopic findings. A total of 88 subjects (48 in the NERD group and 40 in the control group) were included in the study for observation and analysis.

\section{Endoscopy equipment and procedures}

All procedures were performed with a high-definition GFL590WR endoscope that was part of a LASEREO endoscopic system (Fujifilm Co., Tokyo, Japan). Each patient underwent esophageal examination for at least 1 minute using the whitelight mode; immediately thereafter, they were examined using the $\mathrm{LCl}$ mode for a further 1 minute or more. An experienced endoscopist (who had performed more than 1000 examinations using $\mathrm{LCl}$ technology) performed the WLE and $\mathrm{LCl}$ endoscopies in all subjects and digitally recorded conventional WLE images and the corresponding $\mathrm{LCl}$ images of the esophagogastric (EG) junction using USB flash drives.

All endoscopic images were qualified according to resolution and comparability with counterparts. We then added three sets of photographs to two USB flash drives. One drive (drive 1) contained only conventional white-light images (WLI), and the other drive (drive 2) contained conventional white-light and corresponding $\mathrm{LCl}$ images shown together. All the names and dates were removed from the images.

\section{Endoscopic findings of MCE}

MCE was defined as areas of erythema, blurring of the Z-line, friability, decreased vascularity, white turbid discoloration, and edema or accentuation of the mucosal folds $[3,5,12]$. Five participating endoscopists with different levels of endoscopic experience were instructed beforehand to look for distal esophageal mucosal minimal changes using previously described minimal change findings, and some training examples were provided.

\section{Image evaluation}

First, the same experienced endoscopist and two other experienced endoscopists evaluated color patterns for minimal change in 88 pairs of collected WLI and WLI combined with LCI images in both groups, comparing the proportion of minimal change between the two groups. If more than one of the three endoscopists defined a given esophagus as having minimal change, the result was defined as $\mathrm{MCE}(+)$; all other results were defined as MCE (-).

Second, five blinded endoscopists, each with different levels of endoscopic experience, evaluated presence or absence of minimal change using conventional endoscopic images of the 


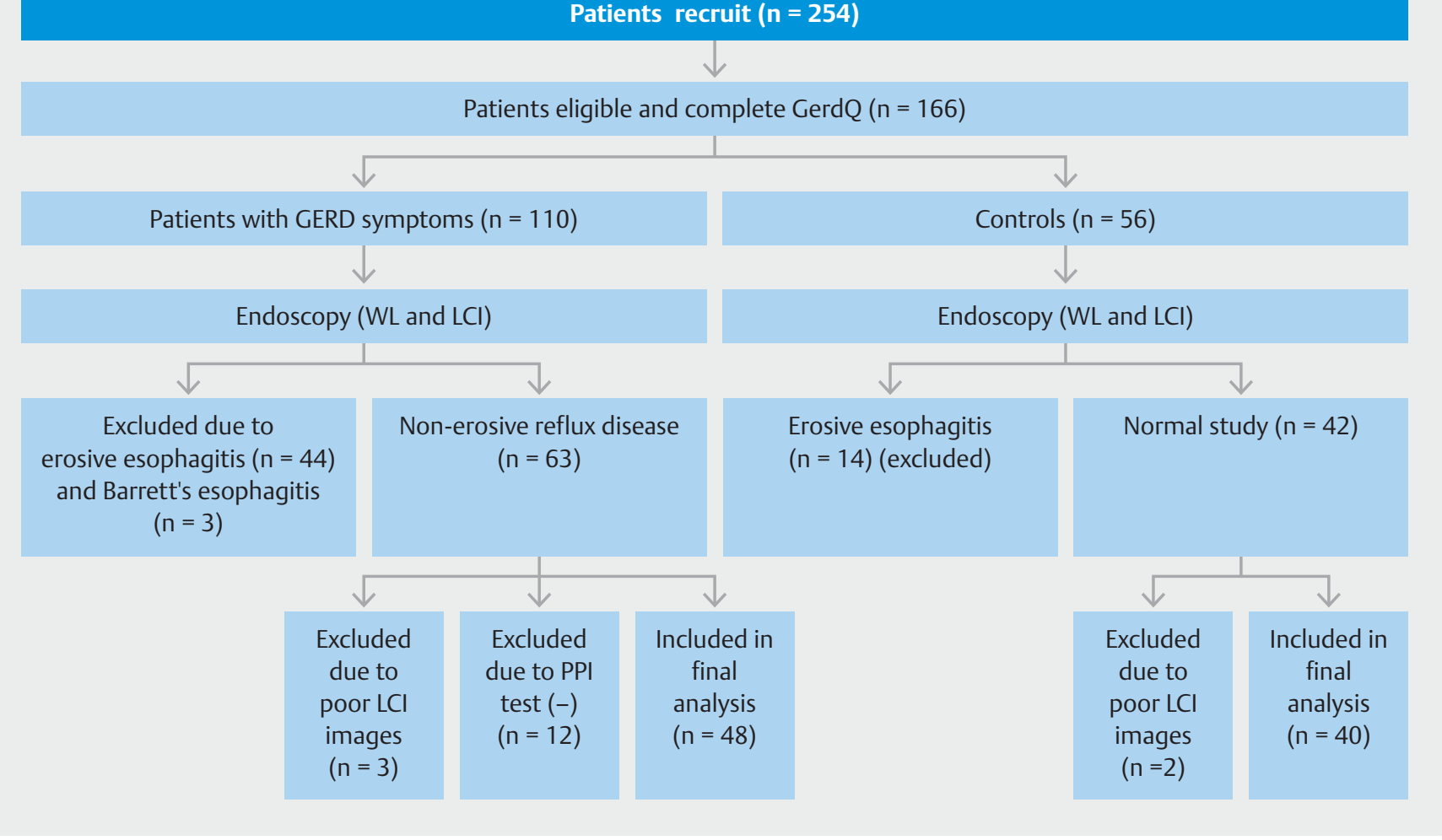

- Fig. 1 Study flow diagram.

esophagogastric junction (EGJ) in both groups and the WLI combined with $\mathrm{LCl}$ images separately. Interval observation using the two drives lasted 2 weeks. A total of 264 images were presented in random groups of three using a USB reader program and were randomly displayed at 15 -second intervals on a personal computer screen. Presence or absence of minimal change was evaluated based on the definition of MCE $(+)$ and MCE (-). The endoscopists were not permitted to review or classify the image again nor were they informed that there would be repeated images on each drive. None of the five endoscopists were given any clinical information and they were unaware of each other's assessments. We followed the method described by Lee [13].

\section{Statistical analysis and sample size}

Data were analyzed using SPSS 21.0 software. Proportions of MCE between the control group and NERD group were compared using the chi-square test. The McNemar test was used to compare detection rates of MCE between the WLI group and the WLI combined with $\mathrm{LCl}$ group. The intra- and interobserver variation for endoscopic diagnosis of MCE by the five endoscopists using WLI or WLI combined with LCI images was analyzed using Kappa statistics. To compute these к coefficients and corresponding $95 \%$ confidence intervals (Cls), we followed the method described by Lee. Strength of agreement was defined as follows: $\mathrm{k}$ values greater than 0.8 denoted excellent agreement, values ranging from 0.8 to 0.6 denoted good agreement, values ranging from 0.6 to 0.4 denoted fair agree- ment, and values less than 0.4 denoted poor agreement [14]. For all tests, $P<0.05$ indicated statistical significance.

For the sample size calculation, based on previous literature [15-18] and previous clinical experience, we used the detection rate as the main index, with a difference value between the detection rates of two types of detection methods of $20 \%$, and set 0.05 and 0.8 as the significance level and power, respectively. Finally, we calculated the estimated number of required cases to be 77 . The final number of patients was 84 by considering the possibility of a certain amount of loss. SAS 9.2 software was used to estimate the sample size.

\section{Results}

\section{Clinical characteristics of patients}

A total of 254 consecutive patients consented to participate in our study, 166 of whom withdrew, including 88 who were excluded due to not completing the GerdQ questionnaire, 58 who had erosive lesions, 3 who had Barrett's esophagitis, and 17 who were excluded due to negative PPI test performance and poor images. The remaining 88 patients were included in the study ( $\triangleright$ Fig.1). Baseline patient characteristics included age, gender, height, and body mass index (BMI). Forty-eight of the 88 patients (54.5\%) were male, and the mean age of our study population was $46.5 \pm 12.9$ years. No significant differences were found between the two groups with regard to demographic features $(P>0.05)$. 


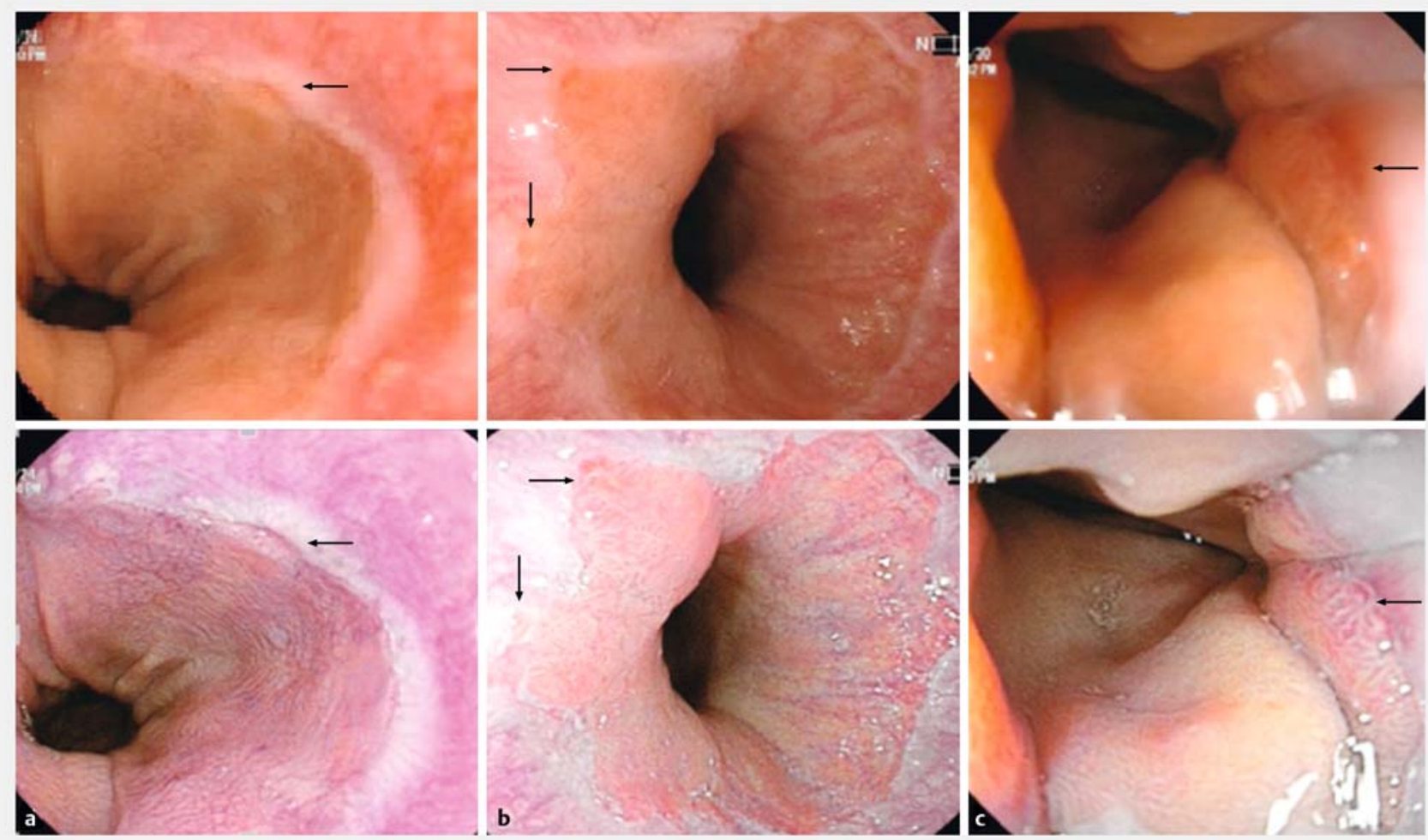

- Fig. 2 Endoscopic image of edema or accentuation of the mucosal folds. A Reddish mucosal change with circular erosion was noted at the squamocolumnar junction (arrow). a The lesion is easily recognizable on link color imaging. $\mathbf{b}$ Reddish mucosal change with edema was noted (arrow). The lesion is easily recognizable as a bright reddish area mixed with purple color on link color imaging. c A prominent gastric mucosal fold was noted at the squamocolumnar junction (arrow). The lesion is easily recognizable as red areas appear redder on link color imaging.

\section{Endoscopic findings of MCE in $\mathrm{LCl}$ compared with WLI}

Minimal changes, such as whitish turbidity and erythema, that were detected using conventional WLE images were more easily recognizable in $\mathrm{LCl}$ in the present $\mathrm{LCl}$ mode, as the white areas appeared whiter and the red areas appeared redder ( Fig.2).

\section{Comparison of MCE with and without use of $\mathrm{LCl}$}

Among the 88 patients, in 44, both WLI and LCI had concordant results, showing normal mucosa. In 28 patients, both techniques were concordant in showing MCE. In 15 patients whose results were normal based on imaging without $\mathrm{LCI}, \mathrm{MCE}$ was observed using $\mathrm{LCl}$. One patient who had been diagnosed as having MCE using imaging without $\mathrm{LCl}$ was diagnosed as normal with $\mathrm{LCl}(P<0.001$, McNemar test) ( $>$ Table 1). The MCE detection rate using $\mathrm{LCl}(43 / 88,48.9 \%)$ was higher than that without $\operatorname{LCl}(29 / 88,33.0 \%, P<0.001)$. Compared with WLE, MCE was easier to detect using imaging combined with $\mathrm{LCl}$.

\section{Comparison of MCE between the NERD group and the Control group using WLI combined with LCl}

The proportion of minimal change in the NERD group $(70.8 \%$, $34 / 48)$ was higher than that in the control group $(22.5 \%, 9 / 40$, $P<0.001)(\triangleright$ Table 2).
- Table 1 Comparative analysis of endoscopic assessment for presence of MCE with and without use of LCI.

\begin{tabular}{|l|l|l|l|}
\hline MCE & $\begin{array}{l}\text { Positive } \\
\text { on WLI }\end{array}$ & $\begin{array}{l}\text { Negative } \\
\text { on WLI }\end{array}$ & P value \\
\hline Positive on $(\mathrm{WLI}+\mathrm{LCl})$ & 28 & 15 & $<.001$ \\
\hline Negative on $(\mathrm{WLI}+\mathrm{LCl})$ & 1 & 44 & \\
\hline $\begin{array}{l}\text { MCE, minimal change esophagitis; LCI, linked color image; WLI, white-light } \\
\text { images. } \mathrm{X}^{2}=12.25, P<0.001\end{array}$ \\
\hline
\end{tabular}

- Table 2 Comparison of MCE between NERD group and control group by using WLI with LCl.

\begin{tabular}{|l|l|l|l|}
\hline MCE & $\begin{array}{l}\text { Positive on } \\
\text { WLI+ LCl }\end{array}$ & $\begin{array}{l}\text { Negative on } \\
\text { WLI+ LCI }\end{array}$ & P value \\
\hline NERD group & 34 & 14 & $<.001$ \\
\hline Control group & 9 & 31 & \\
\hline $\begin{array}{l}\text { MCE, minimal change esophagitis; NERD, non-erosive reflux disease; WLI, } \\
\text { white-light images; LCl linked color image. }\end{array}$ \\
\hline
\end{tabular}


- Table 3 Intraobserver variability for endoscopic diagnosis of MCE between conventional WLI and WLI combined with LCI among 5 endoscopists and weighted pairwise kappa statistics $(95 \% \mathrm{Cl})$.

\begin{tabular}{|l|l|l|l|l|l|}
\hline Endoscopist & A & B & C & D & E \\
\hline $\begin{array}{l}\text { Kappa values }(95 \% \mathrm{CI}) \\
\text { for WLI }\end{array}$ & $\begin{array}{l}0.477 \\
(0.270-0.675)^{1}\end{array}$ & $\begin{array}{l}0.376 \\
(0.154-0.601)^{1}\end{array}$ & $\begin{array}{l}0.543 \\
(0.333-0.709)^{1}\end{array}$ & $\begin{array}{l}0.482 \\
(0.254-0.714)^{1}\end{array}$ & $\begin{array}{l}0.316 \\
(0.083-0.534)^{1}\end{array}$ \\
\hline $\begin{array}{l}\text { Kappa values }(95 \% \mathrm{Cl}) \\
\text { for WLI with LCI }\end{array}$ & $\begin{array}{l}0.477 \\
(0.243-0.666)^{1}\end{array}$ & $\begin{array}{l}0.564 \\
(0.377-0.727)^{1}\end{array}$ & $\begin{array}{l}0.657 \\
(0.494-0.813)^{1}\end{array}$ & $\begin{array}{l}0.726 \\
(0.580-0.863)^{1}\end{array}$ & 0.635 \\
$(0.452-0.795)^{1}$
\end{tabular}

\section{Intraobserver reproducibility}

Based on presence or absence of minimal change, the intraobserver reproducibility levels using conventional endoscopic images and $\mathrm{LCl}$ images are shown in $>$ Table 3. Kappa values and $95 \% \mathrm{Cls}$ for all the endoscopists in diagnosing the 88 cases are also shown in $\$$ Table 3 . With only conventional illumination, intraobserver reproducibility levels were fair for $A, C$ and $\mathrm{D}$ and poor for $\mathrm{B}$ and $\mathrm{E}$. When WLI was combined with $\mathrm{LCl}$, reproducibility levels were fair for $A$ and $B$ and good for $C$, D and $E$. The results showed that the intraobserver reproducibility levels when WLI was combined with LCI were better than those for WLI alone.

\section{Interobserver agreement}

With regard to interobserver agreement, - Table 4 shows the observed agreements between the nine pairs of observers performing endoscopic diagnosis of minimal change, along with the corresponding kappa values and $95 \% \mathrm{Cls}$. When using only conventional endoscopy, except for one pair (B to E) that was poor, kappa values showed moderate to good agreement for the rest of the pairs $(k=0.426$ to 0.705$)$. With respect to WLI combined with $\mathrm{LCl}$, all kappa values were above 0.8 , reflecting excellent interobserver agreement. The results indicated that the combined approach significantly improved agreement $(P<$ $0.001)$.

\section{Discussion}

Currently, endoscopic features of MCE are described by most experts as erythema, blurring of the Z-line, friability, decreased vascularity, white turbid discoloration, and edema or accentuation of the mucosal folds $[3,5,12]$. However, agreement between inexperienced endoscopists is poor for recognition of minimal changes but good for recognition of mucosal breaks [3]; In our study, WLI combined with $\mathrm{LCl}$ was an effective tool for detection of MCE. Therefore, it will improve detection and differentiation of subtle mucosal changes, be beneficial for targeted biopsy of the suspicious area, and may improve detection of intestinal metaplasia and early gastric cancer. Our data showed that compared with WLE, LCI made MCE easier to detect. Intraobserver reproducibility levels using WLI combined with $\mathrm{LCl}$ were better than those using only WLI. When WLI was combined with $\mathrm{LCl}, \mathrm{LCl}$ improved interobserver consistency in MCE diagnosis, and intraobserver and interobserver agreement improved significantly. Meanwhile, prevalence of MCE in patients in the NERD group (70.8\%) was significantly higher than

- Table 4 Interobserver variability for endoscopic diagnosis of MCE between conventional WLI and conventional WLI combined with LCI among 5 endoscopists and weighted pairwise kappa statistics $(95 \% \mathrm{Cl})$.

\begin{tabular}{|c|c|c|c|c|}
\hline Endoscopic & Kappa (WLI) & $95 \% \mathrm{Cl}$ & Kappa (WLI+ LCI) & $95 \% \mathrm{Cl}$ \\
\hline A to $B$ & $0.426^{1}$ & $0.272-0.567^{1}$ & $0.863^{1}$ & $0.779-0.932^{1}$ \\
\hline A to $C$ & $0.541^{1}$ & $0.410-0.680^{1}$ & $0.909^{1}$ & $0.839-0.966^{1}$ \\
\hline A to $D$ & $0.511^{1}$ & $0.352-0.657^{1}$ & $0.874^{1}$ & $0.794-0.942^{1}$ \\
\hline A to $E$ & $0.705^{1}$ & $0.581-0.825^{1}$ & $0.897^{1}$ & $0.819-0.955^{1}$ \\
\hline B to $C$ & $0.642^{1}$ & $0.504-0.765^{1}$ & $0.908^{1}$ & $0.838-0.966^{1}$ \\
\hline B to $D$ & $0.587^{1}$ & $0.439-0.723^{1}$ & $0.897^{1}$ & $0.818-0.954^{1}$ \\
\hline$B$ to $E$ & $0.375^{1}$ & $0.214-0.525^{1}$ & $0.897^{1}$ & $0.827-0.954^{1}$ \\
\hline$C$ to $D$ & $0.577^{1}$ & $0.425-0.715^{1}$ & $0.920^{1}$ & $0.861-0.977^{1}$ \\
\hline$C$ to $E$ & $0.493^{1}$ & $0.343-0.635^{1}$ & $0.920^{1}$ & $0.856-0.966^{1}$ \\
\hline
\end{tabular}


that in the control group. $\mathrm{LCl}$ is a new modality for endoscopy that creates clear and bright endoscopic images using shortwavelength narrow-band laser light combined with white laser light on the basis of BLI technology [8]. LCI makes red areas appear redder and white areas appear whiter, improving the viewer's capacity to recognize slight differences in mucosal color. In the current study, the three experienced endoscopists observed MCE by comparing images with and without $\mathrm{LCl}$, and the results showed a detection rate as high as $48.9 \%$ in the $\mathrm{LCI}$ group, which was obviously better than the rate of detection without $\mathrm{LCl}(33.0 \%)$. Improvement in the detection rates resulted from use of the $\mathrm{LCl}$ technology, which often generates a bright field of view and can produce differences in color contrast in surrounding vessels, enabling easier detection of minimal changes in evaluation of the distal esophagus area. Previous studies also showed that IEE technologies, such as NBI, FICE and i-Scan, improved detection rates for esophageal injury $[4,18-20]$. However, those technologies also have some limitations. For example, with NBI, most endoscopists make a diagnosis of MCE according to presence of increased numbers of intrapapillary capillary loops (IPCLs) and dilated IPCLs [4], which need to be magnified above standard levels and focused closer to the mucosa than with the white-light system, a procedure that can be time-consuming and quite complicated. $\mathrm{LCl}$ can rapidly detect minimal changes without magnification, with color contrast used instead, resulting in reduced operating times and reduced patient pain; the technology is suitable for most endoscopists, including beginners, resulting in good operability.

Our study showed that intraobserver reproducibility and interobserver agreement using WLI combined with LCI were better than those using WLI alone. These differences may be due to several reasons. First, in our study, we found that most minimal changes appeared red under WLI, whereas the changes under $\mathrm{LCl}$ appeared as a more intense red color than the surrounding mucosa because $\mathrm{LCl}$ images are bright, and the red color is adjusted to make the lesions easily identifiable under endoscopy [21]. LCl also clarifies and intensifies white light and provides color images, in contrast to the monochrome images produced by conventional image-enhanced endoscopy, thus improving intra- and interobserver consistency. Second, all investigators had previously agreed upon established criteria for LCI images and were provided with some training examples before the blinded evaluation, improving recognition of MCE. However, in previous studies, levels of interobserver agreement for endoscopic diagnosis of MCE using digital chromoendoscopy were different. For example, both FICE and pCLE have good operating characteristics and can facilitate diagnosis of minimal change esophageal reflux disease (MERD). However, among different observers, pCLE is more consistent on MERD diagnosis [22]. However, there are significant limitations regarding its practical use due to the complex manipulations involved, its poor feasibility, and its high cost. In using $\mathrm{LCl}$ in clinical practice, we found that $\mathrm{LCl}$ improved visualization of the EG], improving intraobserver and interobserver agreements and allowing for easy recognition of standardized, simple and precise endoscopic reading criteria of minimal mucosal changes, re- gardless of whether the evaluators were trainees or experienced endoscopists.

Our study also has limitations. First, because it was a singlecenter study, the sample size was small. A larger sample size is required to provide statistical significance for the MCE detection rate. Second, the diagnostic criteria of NERD based on GERD symptoms may have led to formation of heterogeneous groups with other gastrointestinal functional diseases. Therefore, the patients in our study may have been subjected to a selection bias toward recruitment of more functional heartburn patients. Because we did not perform confirmatory testing (i. e., 24-hour pH-metry), asymptomatic GERD and functional heartburn patients may have been included as controls and cases, respectively. However, this disadvantage was partially overcome in our study by performing a PPI test instead, thus minimizing the probability of including patients with functional heartburn $[23,24]$. Third, no histological evaluations of MCE were conducted. Histology is the gold standard for reflux disease diagnosis, and choice of biopsy site is very important. Variability in biopsy sites is likely to influence study outcomes; meanwhile, lack of a standardized approach to histological assessment makes direct comparisons between studies somewhat uncertain and hampers characterization of histological changes for routine diagnostic purposes [25]. With regard to the endoscopic description of MCE, based on current research, there are no clear guidelines, such as the Los Angeles Classification; therefore, endoscopists are more subjective in the diagnosis of MCE, and discrepancies may occur.

\section{Conclusion}

In conclusion, this prospective, single-center study revealed that WLE combined with LCl was significantly superior to conventional WLE alone in detecting MCE. Intraobserver reproducibility and interobserver agreement in MCE detection were improved when $\mathrm{LCI}$ was applied with conventional imaging. Therefore, $\mathrm{LCl}$ may be used as a better IEE endoscopic technique for detecting MCE.

\section{Competing interests}

None

References

[1] Vakil N, van Zanten SV, Kahrilas P et al. The Montreal definition and classification of gastroesophageal reflux disease: a global evidencebased consensus. Am J Gastroenterol 2006; 101: 1900-1920

[2] Nakamura T, Shirakawa K, Masuyama H et al. Minimal change esophagitis's disease with characteristic differences to erosive oesophagitis. Aliment Pharmacol Ther 2005; 212: (Suppl. 01): 19-26

[3] Armstrong D, Bennett JR, Blum A et al. The endoscopic assessment of esophagitis: a progress report on observer agreement. Gastroenterology 1996; 111: 85-92 
[4] Sharma P, Wani S, Bansal A et al. A feasibility trial of narrow band imaging endoscopy in patients with gastroesophageal reflux disease. Gastroenterology 2007; 133: $454-464$

[5] Hongo M. Minimal changes in reflux esophagitis: red ones and white ones. J Gastroenterol 2006; 41: 95 -99

[6] Miwa H, Yokoyama T, Hori K et al. Interobserver agreement in endoscopic evaluation of reflux esophagitis using a modified Los Angeles classification incorporating grades $\mathrm{N}$ and $\mathrm{M}$ : a validation study in a cohort of Japanese endoscopists. Dis Esophagus 2008; 21: 355 - 363

[7] Min M, Deng P, Zhang W et al. Comparison of linked color imaging and white-light colonoscopy for detection of colorectal polyps: a multicenter, randomized, crossover trial. Gastrointest Endosc 2017; 86: $724-730$

[8] Okada M, Sakamoto H, Takezawa T et al. Laterally spreading tumor of the rectum delineated by linked color imaging technology. Clin Endosc 2016; 49: 207 - 208

[9] Osawa H, Yamamoto $\mathrm{H}$. Present and future status of flexible spectral imaging color enhancement and blue laser imaging technology. Dig Endosc 2014; 26: (Suppl. 01): 105-115

[10] Jones R, Junghard O, Dent J et al. Development of the GerdQ, a tool for the diagnosis and management of gastro-oesophageal reflux disease in primary care. Aliment 2009; 30: 1030-1038

[11] Kim JB, Shin SR, Shin WG et al. Prevalence of minimal change lesions in patients with non-erosive reflux disease: a case-control study. Digestion 2012; 85: $288-294$

[12] Kim JH, Park H, Lee YC et al. Is minimal change esophagitis really part of the spectrum of endoscopic findings of gastroesophageal reflux disease? A prospective, multicenter study Endoscopy 2011; 43: 190 195

[13] Lee YC, Lin JT, Chiu HM et al. Intraobserver and interobserver consistency for grading esophagitis with narrow-band imaging. Gastrointest Endosc 2007; 66: 230-236

[14] Landis JR, Koch GG. The measurement of observer agreement for categorical data. Biometrics 1977; 33: 159-174
[15] Yoshikawa I, Yamasaki M, Yamasaki T et al. Lugol chromoendoscopy as a diagnostic tool in so-called endoscopy-negative GERD. Gastrointest Endosc 2005; 62: 698 - 703

[16] Falk GW. Is conventional endoscopic identification of non-erosive reflux disease adequate? Digestion 2008; 78: (Suppl. 01): 17-23

[17] Gomes Jr CA, Loução TS, Carpi G et al. A study on the diagnosis of minimal endoscopic lesions in nonerosive reflux esophagitis using computed virtual chromoendoscopy (FICE). Arq Gastroenterol 2011; 48: $167-170$

[18] Fock KM, Teo EK, Ang TL et al. The utility of narrow band imaging in improving the endoscopic diagnosis of gastroesophageal reflux disease. Clin Gastroenterol Hepatol 2009; 7: 54 - 59

[19] Kodashima S, Fujishiro M. Novel image-enhanced endoscopy with i-scan technology. World J Gastroenterol 2010; 16: 1043-1049

[20] Netinatsunton N, Sottisuporn ], Attasaranya S et al. i-Scan detection of minimal change esophagitis in dyspeptic patients with or without Gastroesophageal Reflux disease. BMC Gastroenterol 2016; 16: 4

[21] Sun X, Dong T, Bi Y et al. Linked color imaging application for improving the endoscopic diagnosis accuracy: a pilot study. Sci Rep 2016; 6: 33473

[22] Pittayanon R, Aumkaew S, Rerknimitr E et al. Flexible spectral imaging color enhancement and probe-based confocal laser endomicroscopy in minimal change esophageal reflux disease. Korean J Gastroenterol 2016; 68: 29-35

[23] Fass R, Ofman JJ, Gralnek IM et al. Clinical and economic assessment of the omeprazole test in patients with symptoms suggestive of gastroesophageal reflux disease. Arch Intern Med 1999; 159: 2161 2168

[24] Gasiorowska A, Fass R. The proton pump inhibitor test in GERD: does it still have a role? J Clin Gastroenterol 2008; 42: 867 - 874

[25] Dent ]. Microscopic esophageal mucosal injury in nonerosive reflux disease. Clin Gastroenterol Hepatol 2007; 5: 4-16 